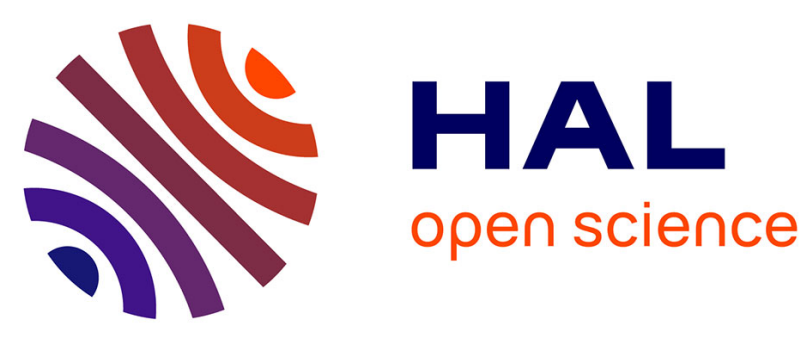

\title{
Sur le fluage par déviation
}

\author{
J. Friedel
}

\section{To cite this version:}

J. Friedel. Sur le fluage par déviation. Revue de Physique Appliquée, 1977, 12 (10), pp.1649-1654. 10.1051/rphysap:0197700120100164900 . jpa-00244384

\section{HAL Id: jpa-00244384 https://hal.science/jpa-00244384}

Submitted on 1 Jan 1977

HAL is a multi-disciplinary open access archive for the deposit and dissemination of scientific research documents, whether they are published or not. The documents may come from teaching and research institutions in France or abroad, or from public or private research centers.
L'archive ouverte pluridisciplinaire HAL, est destinée au dépôt et à la diffusion de documents scientifiques de niveau recherche, publiés ou non, émanant des établissements d'enseignement et de recherche français ou étrangers, des laboratoires publics ou privés. 
Classification
Physics Abstracts
62.20

\title{
SUR LE FLUAGE PAR DÉVIATION
}

\author{
J. FRIEDEL \\ Laboratoire de Physique des Solides, Bâtiment 510, Université Paris-Sud, 91405 Orsay, France
}

(Reçu le 19 arril 1977, accepté le 27 juin 1977)

\begin{abstract}
Résumé. - On analyse les processus de déviation qui peuvent être mis en jeu dans le fluage à moyennes températures des métaux cubiques à faces centrées.
\end{abstract}

\begin{abstract}
The possible role of crosslipping is discussed in the creep at moderate temperatures of face centred cubic metals.
\end{abstract}

1. Introduction. - Il est classique [1] de ranger les déformations thermiquement activées des métaux purs cubiques à faces centrées en quatre classes, d'énergies d'activation différentes

- glissement contre la friction de réseau, avec formation de décrochements $\left(U_{1}\right)$,

- glissement avec traversée de la forêt, avec formation de crans $\left(U_{2}\right)$,

- glissement dévié $\left(U_{3}\right)$,

- montée par diffusion $\left(U_{4}\right)$.

Les expériences de Dorn [2] montrent que, dans l'aluminium, $U_{1}$ et $U_{2}<U_{3}<U_{4}$, donc que ces divers processus limitent successivement la vitesse de déformation plastique à température croissante. Ce fait semble assez général dans les métaux cubiques à faces centrées, où les processus de déviation dominent le stade III de la déformation $\sigma(\varepsilon)$ à température $T$ constante et les fluages $\varepsilon(t)$ à fortes contraintes $\sigma$ dans le domaine des températures moyennes [1]. Ce sont aussi ces processus de déviation qui semblent limiter le laminage à chaud des aciers austénitiques.

On sait par ailleurs que le développement du glissement dévié provoque un réarrangement des dislocations en cellules polygonisées dont l'arrangement peut, quand les déviations sont faciles, devenir presque aussi parfait que dans les déformations par diffusion [1].

Le passage du stade II au stade III dans les courbes $\sigma(\varepsilon, T)$ a donné lieu aux premières analyses de l'activation thermique de la déviation [3]. Il a suscité de nombreuses études théoriques et expérimentales $[4,5]$.

Peu d'études portent par contre sur le détail des processus mis en jeu quand la polygonisation est bien établie, par exemple dans le stade de fluage linéaire. Il semble même que la possibilité de fluage par déviation ait été oubliée pendant une décennie.

Deux études récentes reposent le problème.

1) Dans un article récent désumant ses travaux, Myshlyaev [7] montre le développement de la polygonisation lors du fluage à température modérée, sous fortes contraintes. Il obtient une loi de fluage avec une énergie d'activation dépendant de la contrainte appliquée $\sigma$ :

$$
U=U_{0}-\sigma v .
$$

Il trouve $U_{0}<U_{4}$, énergie d'activation de la diffusion, et

$$
v \simeq \alpha b^{2} h,
$$

où $b$ est le vecteur de Burgers, $h$ la longueur moyenne des arcs libres de dislocations dans les parois et $\alpha$ un facteur numérique peu supérieur à l'unité.

Myshlyaev déduit de ces observations que le processus mis en jeu est la création thermiquement activée de crans [7], lors de la destruction des parois de dislocation, par traversée mutuelle de deux brins C, $C^{\prime}$ (Fig. 1a). La libération d'un tel brin augmente localement la longueur $h$ dans $v$, relation (2), donc réduit $U:$ les brins voisins peuvent se libérer plus facilement, et le processus peut devenir catastrophique (Fig. 1b), et aboutir à la destruction de la paroi. Myshlyaev pense que ce sont les parois les moins stables où ce processus commence; et, une fois mis en route, il peut conduire à une réorgani- 


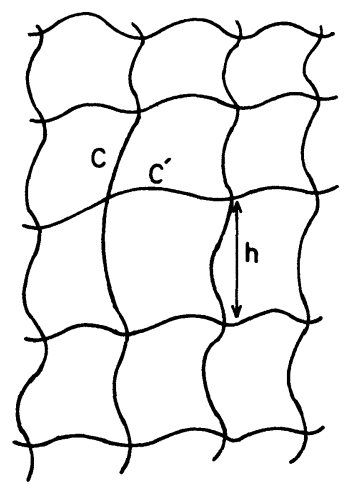

a.

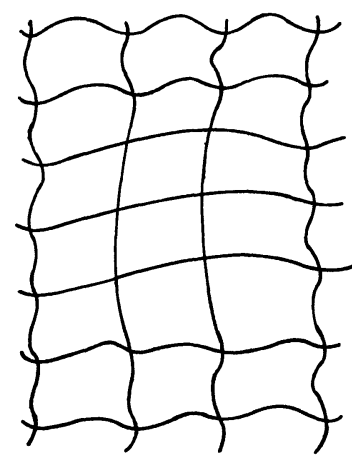

b.
Fig. 1. - Destruction d'un sous-joint par croisement.

[Destruction of a subboundary by dislocation crossing.]

sation locale importante des cellules par glissement. Le processus, en libérant des dislocations qui vont former ou renforcer d'autres parois après glissement, conduit finalement au fluage.

Dans des cas comme l'aluminium, on sait [8] cependant que la portion thermique de la traversée de la forêt est éteinte dès $100 \mathrm{~K}$. Dans les processus de glissement un peu au-dessus de la température ambiante des métaux CFC étudiés par Myshlyaev, on est donc à peu près sûr que c'est la déviation qui limite la vitesse. D'ailleurs les valeurs qu'il donne pour $U_{0}$ sont du bon ordre de grandeur pour la déviation $U_{3}$, et généralement trop fortes pour la formation de crans $U_{2}$ par traversée de la forêt [2].

2) Récemment, Martin [8] a observé en microscopie électronique à haute tension des destructions progressives de parois de polygonisation dans des conditions de fluage analogues à celles de Myshlyaev. Si l'on classe les parois d'après leur orientation plutôt de flexion (coin) ou plutôt de torsion (vis), il est intéressant de noter que ce sont ces dernières qui se détruisent. Comme l'a remarqué récemment Poirier [12], cette dernière observation est un critère assez sûr que l'on est en présence de fluage par déviation, et non de fluage par diffusion, où ce seraient les parois de flexion qui évolueraient. Dans les métaux CFC, où la montée se fait à une température supérieure aux déviations, les parois de torsion seraient d'ailleurs pratiquement toutes détruites dans le régime de fluage par diffusion.

Myshlyaev classe les joints de torsion en 3 grandes familles :

- celles faites d'une seule famille de dislocations parallèles,

- celles faites de deux familles de dislocations surtout vis et à peu près orthogonales, dans un plan très grossièrement voisin de (100),

- celles faites de trois familles de dislocations vis formant un réseau hexagonal dans un plan voisin de (111).
Il estime que ce sont les parois de la deuxième famille qui se détruisent.

Martin observe, quant à lui, dans le microscope, lors de telles destructions, des déviations vers le plan de glissement de contrainte résolue maxima, et souvent un plan de glissement exotique [9] ((110) ...).

On est alors amené à se demander comment exactement joue le processus de déviation dans le fluage à température modérée et fortes contraintes :

- est-ce dans la formation ou la destruction des parois de polygonisation, ou dans les deux?

- comment ce processus peut-il conduire aux lois de fluage (1), (2), qui ne sont pas celles du début de stade III?

2. Formation par déviation des parois de polygonisation. - Quand une dislocation vis $\mathrm{V}$ rencontre dans son glissement un arbre A, la constriction formée sur V lors du croisement de l'arbre peut faciliter [5] la germination sur $\mathrm{V}$ d'une déviation dans un autre plan de glissement $\mathbf{P}^{\prime}$ (Fig. 2).

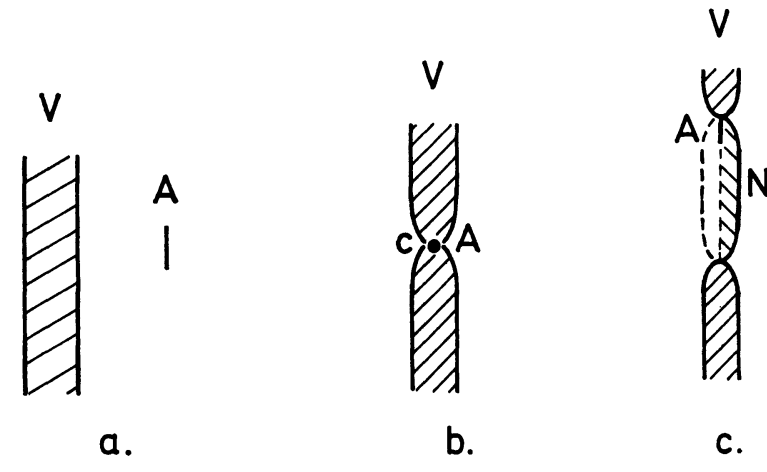

Fig. 2. - Processus de déviation à un croisement.

[Crossslip during crossing.]

Si de plus l'arbre A est dans le plan $\mathrm{P}^{\prime}$, la déviation peut être facilitée si son développement peut réduire l'énergie de ligne [14] :

- si $b_{\mathrm{A}} b_{\mathrm{v}}>0$, la portion déviée de $\mathrm{V}$ peut, par glissement dans $\mathrm{P}^{\prime}$, se placer perpendiculairement à $\mathrm{A}$ (Fig. 3a),

- si $b_{\mathrm{A}} b_{\mathrm{v}}<0$, la déviation peut permettre une meilleure dissociation du nœud quadruple en deux nœuds triples [6] dans $P^{\prime}$ (Fig. 3b).

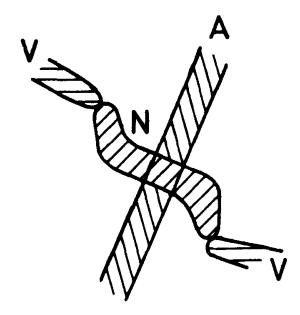

a

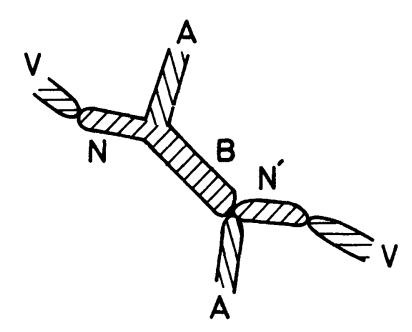

b
Fig. 3. - Réduction d'énergie de ligne par déviation lors d'un croisement.

[Reduction of line energy by crossslip during crossing.] 
Une collection de dislocations $\mathrm{V}$ arrivant sur une collection d'arbres A situés dans le même plan $\mathbf{P}^{\prime}$ ou des plans $\mathbf{P}^{\prime}$ voisins peut ainsi, par une succession de déviations, donner une paroi de rotation à trois ou deux familles de dislocations vis (Fig. 4).

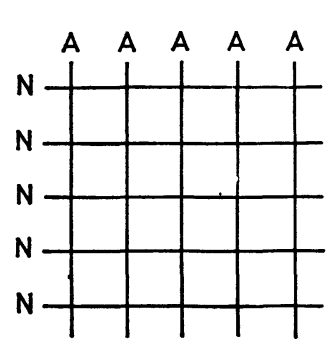

a.

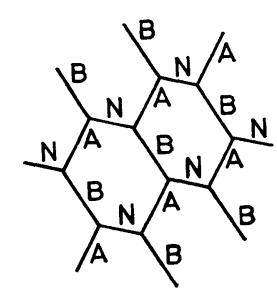

b.
FIG. 4. - Parois de rotation à 3 ou 2 familles de dislocations vis.

[Rotation boundaries with 3 or 2 families of screw dislocations.]

Le processus décrit figures 2 à 4 a été observé au microscope électronique à haute tension [8]. D'après la remarque illustrée figure 3 , les déviations mises en jeu doivent avoir un spectre d'énergies-d'activation systématiquement inférieures à celle $U_{3}$ mesurée au début du stade III. Dans le fluage sous forte contrainte $\sigma$, cette énergie est encore diminuée du travail que peut faire l'arbre $\mathrm{A}$, dans le glissement sous l'effet de $\sigma$ rendu possible par la déviation. Ceci réduit l'énergie de $\sigma v$, où le volume d'activation est donné par (2), si $\alpha b \simeq b$ est la taille du germe critique de déviation.

Finalement, l'absorption des dislocations vis par les joints de torsion et l'élimination des contraintes correspondantes doit se faire avec une énergie d'activation du type (1), (2), où $U_{0}$ a une distribution comprise entre zéro et l'énergie $U_{3}$ de déviation sous contrainte faible et avec une constriction préalable produite par un cran [5].

On peut encore remarquer que les parois hexagonales du type $\mathrm{b}$, figure 4 , semblent plus régulières et plus stables que les parois carrées du type a. Ceci peut être relié au fait que, dans le cas $b, 3$ dislocations vis $A, N, B$ peuvent glisser dans le même plan (III). Dans le cas a au contraire, des dislocations $A$ et $\mathbf{N}$ ne peuvent être orthogonales que dans un plan (100) qui n'est pas leur plan de glissement normal de basse température. Il faut donc admettre, pour la formation des parois du type a, soit que les dislocations dévient dans un plan exotique (100), soit que des déviations successives et compliquées dans plusieurs plans (III) les amènent finalement dans un plan voisin de (100). Le premier processus semble le plus vraisemblable, mais mériterait une étude expérimentale plus approfondie.

3. Destruction par déviation des parois de polygonisation. - Une dislocation vis ne peut s'échapper par glissement d'une paroi de torsion que sous une contrainte élevée. Il y a pour cela deux raisons (cf. appendice) :

a) la contrainte critique d'échappement par glissement est élevée (au moins $\frac{\mu b}{2 h}$, soit typiquement $10^{-2} \mu$, dans le cas le plus instable de familles de vis parallèles),

b) dans la formation de la paroi par déviation, les dislocations vis sont nécessairement dissociées dans le plan faisant l'angle minimum avec le plan de la paroi. Dans ce plan, la contrainte critique est généralement supérieure à sa valeur minimum; et ce plan n'est pas le plan de contrainte résolue maxima.

Si le joint est formé de plus qu'une famille de dislocations vis, on peut alors invoquer la germination d'une déviation au croisement de deux dislocations pour faciliter l'échappement de la dislocation vis.

Pour qu'il y ait échappement, il faut alors que simultanément deux conditions soient satisfaites (Fig. 5) :

1) Germination d'une déviation sous contrainte : la vitesse du processus a une énergie d'activation de forme (1), (2) ou $U_{0}$ est maintenant limitée inférieurement par l'énergie de déviation $U_{3}$. La contrainte $\sigma_{1}$ nécessaire pour obtenir une vitesse constante décroit linéairement à $T$ constante.

2) Échappement de la contrainte de rappel de la paroi $\sigma_{2}$ : elle est minimale près du plan de contrainte résolue maxima, tant que celui-ci fait avec le plan de la paroi un angle notable (typiquement supérieur à $\pi / 6$, cf. appendice).

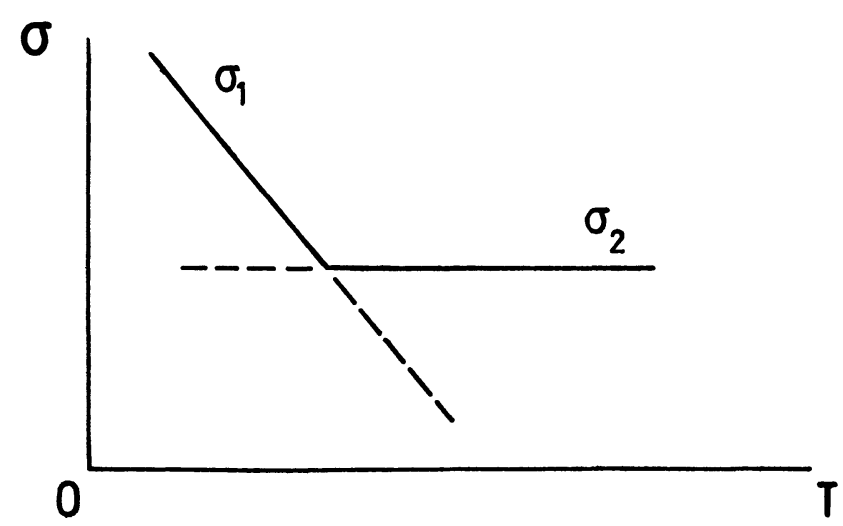

Fig. 5. - Deux régimes de destruction par déviation.

[Two regimes of destruction by crossslip.]

Cette discussion montre que seules les parois assez mal formées et instables peuvent se détruire $\left(\sigma_{2}\right.$ petit); pour ces parois, on s'attend à un processus thermiquement activé $\left(\sigma_{1}\right)$ avec une énergie $U_{0}$ peu supérieure à l'énergie de déviation.

4. Discussion. - L'analyse précédente montre que les parois sont plus faciles à former qu'à détruire. 
Si donc il y a assez de sources intragranulaires pour fournir une déformation appréciable, le fluage doit être principalement limité par l'absorption par les parois déjà formées des dislocations, produites.

Dans le cas contraire, les seules dislocations mobiles peuvent être fournies par la destruction des parois les plus instables.

Les deux processus sont certainement limités par la déviation aux températures modérées et aux fortes contraintes. Ils donnent lieu à des lois d'activation très semblables, du style (1), (2). Le premier est compatible avec une constance dans le temps de la taille des blocs de polygonisation, alors que le second conduit plutôt à un grossissement de la taille des blocs.

Il est vraisemblable que ces deux types de processus coexistent. Mais des expériences plus approfondies sont nécessaires pour départager leurs importances relatives.

L'auteur remercie les $D^{\text {rs }}$ J.-L. Martin, M. M. Myshlyaev et J.-P. Poirier pour les discussions qui ont mené à la rédaction de cette note.

Appendice. - On considère d'abord un sous-joint formé d'une famille de dislocations vis parallèle à l'axe $O z$, équidistantes dans le plan $y O z$ et de vecteur de Burgers $b$ (Fig. A1). On déplace une dislocation D du sous-joint dans un plan de glissement $P$.

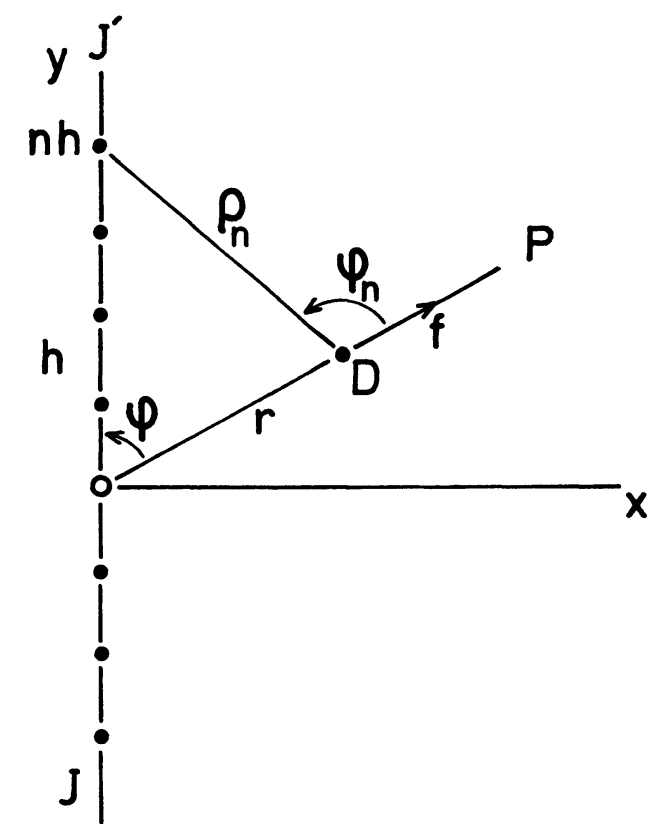

FIG. A1. - Dislocations D glissant hors d'un sous-joint de dislocations vis équidistantes.

[Dislocation D slipping out of a subboundary made of equidistant new dislocations.]

La dislocation en $y=n h$ exerce sur $\mathrm{D}$ une force répulsive dont la projection sur le plan OP est

$$
f_{n}=-\frac{\mu b^{2}}{2 \pi \rho_{n}} \cos \Phi_{n}
$$

La force totale exercée par le sous-joint sur $\mathrm{D}$ et résolue dans le plan $\mathrm{P}$ est

$$
f=\sum_{n \neq 0} f_{n}
$$

Utilisant le fait que

$$
\sum_{n \text { entier }} \delta(y-n h)=\frac{1}{h} \sum_{q \text { entier }} \exp \frac{2 \pi i q y}{h}
$$

on obtient

$$
f=-\frac{\mu b^{2}}{2 \pi r}-\frac{\mu b^{2}}{2 \pi h} \sum_{q \text { entier }} \int_{-\infty}^{\infty} \mathrm{e} \frac{2 \pi i q y}{h} \frac{y \cos \Phi-r}{(y-r \cos \Phi)^{2}+r^{2} \sin ^{2} \Phi} d y
$$


Une intégration par résidus pour $q>0$ et $q<0$ donne [13]

où

$$
f=g_{\mathrm{o}}-\frac{\mu b^{2}}{h}\left[\frac{\sin \Phi}{u}-\frac{\left[\sin (u \cot \Phi+\Phi)-\sin \Phi \mathrm{e}^{-u}\right] \mathrm{e}^{-u}}{1-2 \cos (u \cot \Phi) \mathrm{e}^{-u}+\mathrm{e}^{-2 u}}\right]
$$

$$
u=\frac{2 \pi r \sin \Phi}{h}
$$

et

$$
g_{0}=-\frac{\mu b^{2}}{2 \pi h} \int_{-\infty}^{\infty}\left(y-\frac{y \cos \Phi-r}{r \cos \Phi)^{2}+r^{2} \sin ^{2} \Phi} d y\right.
$$

$g_{\mathrm{o}}$ est la contribution de $f$ pour $q=0$, c'est-à-dire la contrainte due à la distribution continue et uniforme de dislocations infinitésimales de même densité moyenne de vecteurs de Burgers que le sous-joint. C'est la projection dans le plan $\mathbf{P}$ d'une force normale au sous-joint; elle est discontinue de part et d'autre du sous-joint $(x \gtrless 0)$.

Un développement pour $r$ grand donne alors $(r>>h / \sin \Phi)$ :

$$
f \simeq g_{\mathrm{o}}-\frac{\mu b^{2}}{2 \pi r}+O\left(\mathrm{e}^{-\frac{2 \pi r \sin \Phi}{h}}\right)
$$

Un développement pour $r$ petit donne de même

$$
f \simeq g_{\mathrm{o}}-\frac{\mu b^{2}}{2 h} \sin \Phi\left[1-\frac{2 \pi r \sin ^{4} \Phi-\cos ^{4} \Phi}{6 h}+O_{2}\left(\frac{r}{h}\right)\right] .
$$

La figure A2 montre que $\left|f-g_{\mathrm{o}}\right|$ décroît constamment avec $r$ pour $\Phi>\pi / 4$, mais passe par un ou plusieurs maxima pour $\Phi<\pi / 4$. La figure A3 donne la valeur maximale de $\left|f-g_{\mathrm{o}}\right|$ en fonction de l'angle $\Phi$. On voit que cette valeur passe par un minimum égal à $\frac{\mu b^{2}}{2 \sqrt{2 h}}$ pour $\Phi=45^{\circ}$, et qu'elle varie assez peu par rapport à cette valeur pour $\Phi>\frac{\pi}{6}$.
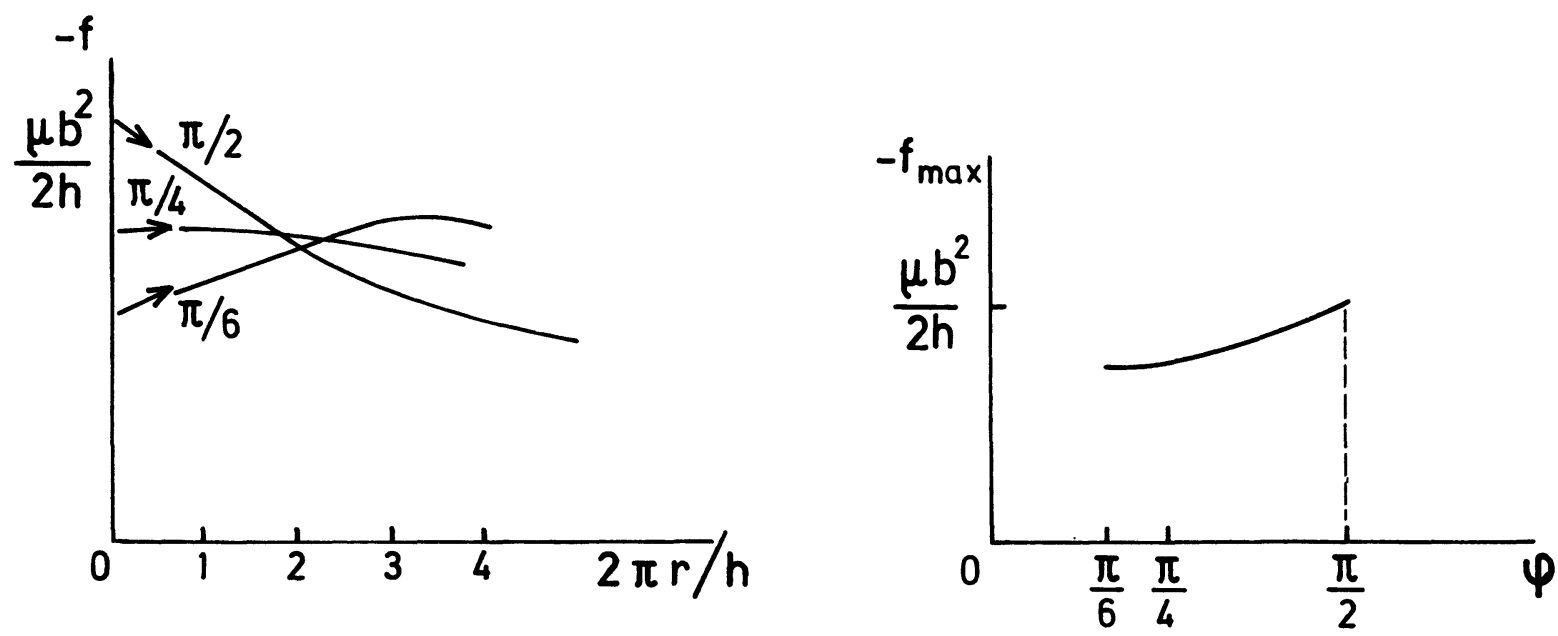

Fig. A2. - Variation de la force de rappel $f$ avec le déplacement pour diverses orientations $\Phi$ du plan de glissement.

[Variation of the force $f$ with displacement $r$ for various orientations $\Phi$ of the slip plane.]

On considère maintenant l'effet sur la dislocation déplacée d'une seconde famille de dislocations vis équidistantes alignées paralièlement à $O y$ dans le plan $y O z$, figure $\mathrm{A} 1$. Un calcul analogue montre que cette seconde famille exerce sur la dislocation placée en $(r, \Phi)$ une force $f^{\prime}$ qui compense $g_{0}$ et produit une correction qui varie périodiquement le long de cette dislocation, avec une période $h^{\prime}$ égale à la distance entre les dislocations de la seconde famille :

$$
f^{\prime}=-g_{\mathrm{o}} \pm \frac{\mu b^{2} \sin \Phi}{h^{\prime}} \frac{\left(\cos \frac{2 \pi z}{h^{\prime}}-\mathrm{e}^{-u^{\prime}}\right) \mathrm{e}^{-u^{\prime}}}{1-2 \cos \frac{2 \pi z}{h^{\prime}} \mathrm{e}^{-u^{\prime}}+\mathrm{e}^{-2 u^{\prime}}}
$$


avec

$$
u^{\prime}=\frac{2 \pi r \sin \Phi}{h^{\prime}}
$$

Il est facile de voir que tant que $h^{\prime} \simeq h$, cette correction $f^{\prime}+g_{\mathrm{o}}$ reste faible vis à vis du terme principal $f-g_{\mathrm{o}}$ aux distances $r$ telles que $u$ et $u^{\prime}$ soient de l'ordre de quelques unités. D'après les résultats de la figure A2, la seconde famille joue assez peu de rôle sur la force d'échappement à grande distance. Elle peut avoir par contre un certain effet au contact, par transformation de points quadruples en 2 points triples si les 2 systèmes ne sont pas parfaitement vis [14].

\section{Bibliographie}

[1] Friedel J., Dislocations (Pergamon Londres) 1964, 258, 264, 277, 305, 311, 315.

[2] Dorn J. E., Contrat NONR 222 (49) séries 103, no 3 (1957).

[3] SchoеcK G., Bristol Conference, Physical Society Londres (1954).

[4] FRIeDel J., ibid. 162.

[5] Escaig B., J. Physique 29 (1968) 225.

[6] Washburn J., Appl. Phys. Lett. 7 (1965) 183.

[7] Myshlyaev M. M., $4^{\text {th }}$ Intern. Conf. on the strength of metals and Alloys, Nancy 1976, under press; aussi Sov. Phys. Solid State 12 (1970) 664; et al. Phys. Stat. Sol. (a) (1971) 325.

[8] MarTin J. L., J. Microsc. Spectrosc. Electron. 1 (1976); MYSH- lyaev M. M., Caillard D. et Martin J. L., $5^{\text {e }}$ Congrès Int. Microscopic Electron. Haute Tension, Kyoto (1977).

[9] Crussard C., Bull. Soc. Fr. Min. 68 (1945) 174; Le Hazrif R., Dorizzi P. et Poirier J. P., Acta Metal. 21 (1973) 903; Edelin G., Scripta Met. 6 (1972) 1185; Caillard D. et Martin J. L., $4^{\mathrm{e}}$ Congrès international de Microscopie électronique, Toulouse (1975).

[10] Cottrell A. H., Dislocations and Plastid Flow (Oxford University Press) 1953.

[11] Friedel J., ibid. 309.

[12] PoIRIER J. P., Revue Phys. Appl. (1977) sous presse.

[13] Li J.C.M. et Needham C. D., J. Appl. Phys. 31 (1960) 1318.

[14] CF Bullough R. et Sharp J. V., Phil. Mag. 11605 (1965) pour une discussion plus détaillée. 